\title{
Schwannoma in the Dorsal Paraspinal Muscle: A Case Report
}

\author{
Yong Guk Kim¹, Tae Wan Kim¹, Min A Kim², Kwan Ho Park ${ }^{1}$ \\ Departments of ${ }^{1}$ Neurosurgery, ${ }^{2}$ Pathology, VHS Medical Center, Seoul, Republic of Korea
}

Corresponding author: Tae Wan Kim Department of Neurosurgery, VHS Medical Center, 53, Jinhwangdo-ro 61-gil, Gangdong-gu, Seoul 05368, Republic of Korea

Tel: $+82-2-2225-1363$

Fax: $+82-2-2225-4152$

E-mail: euro3399@naver.com

Received: June 3, 2020

Revised: June 22, 2020

Accepted: June 29, 2020
A schwannoma is a tumor arising from Schwann cells of the nerve sheath, and the majority of schwannomas are slow-growing. An intramuscular schwannoma is extremely rare and a preoperative diagnosis is very difficult to obtain due to their low incidences and ambiguous clinical signs and symptoms. A 79-year-old man presented with a slow-growing mass with tenderness on the right side of his back. A magnetic resonance imaging (MRI) scan revealed a well-demarcated oval-shaped mass lesion in the right multifidus muscle at the L5 level measuring $2.0 \times 2.0 \times 3.5 \mathrm{~cm}$. Complete surgical excision of the lesion was performed. When a patient has a palpable, intramuscular back mass, an MRI should be performed to assist in obtaining a diagnosis. Although rare, schwannoma should be considered in the differential diagnosis of well-defined, oval-shaped soft tissue masses arising within the paraspinal muscles.

Key Words: Magnetic resonance imaging; Neurilemmoma; Paraspinal muscles

\section{INTRODUCTION}

A schwannoma is a benign, encapsulated peripheral nerve sheath tumor. An intramuscular schwannoma is extremely rare and grows slowly. The symptoms of an intramuscular schwannoma are dependent upon the site of origin. In most cases, the symptoms are ambiguous, making a preoperative diagnosis difficult. Further, specific motor weakness due to motor branch involvement is uncommon ${ }^{10)}$. We report a rare case of schwannoma in the dorsal paraspinal muscles.

\section{CASE REPORT}

A 79-year-old man was admitted to the hospital because of tenderness on the right side of his back. A palpable mass was identified 6 years prior to his admittance. No neurological deficits were detected, and the laboratory data were within normal limits; the patients' medical history was unremarkable.

The mass was observed in the right multifidus muscles at the $\mathrm{L} 5$ level using magnetic resonance imaging (MRI). The MRI scan showed an oval-shaped tumor measuring $2.0 \times 2.0 \times 3.5$ $\mathrm{cm}$. There was a well-defined and heterogeneous intermediate signal on the axial T1-weighted image (WI), and a strong enhancement of central mass lesion was noted (Fig. 1). The preoperative diagnostic evaluation suggested that the growth was a metastatic, sarcoma, or a peripheral nerve sheath tumor.

The patient underwent surgery, and the mass was completely excised. The mass was pinkish and well encapsulated with
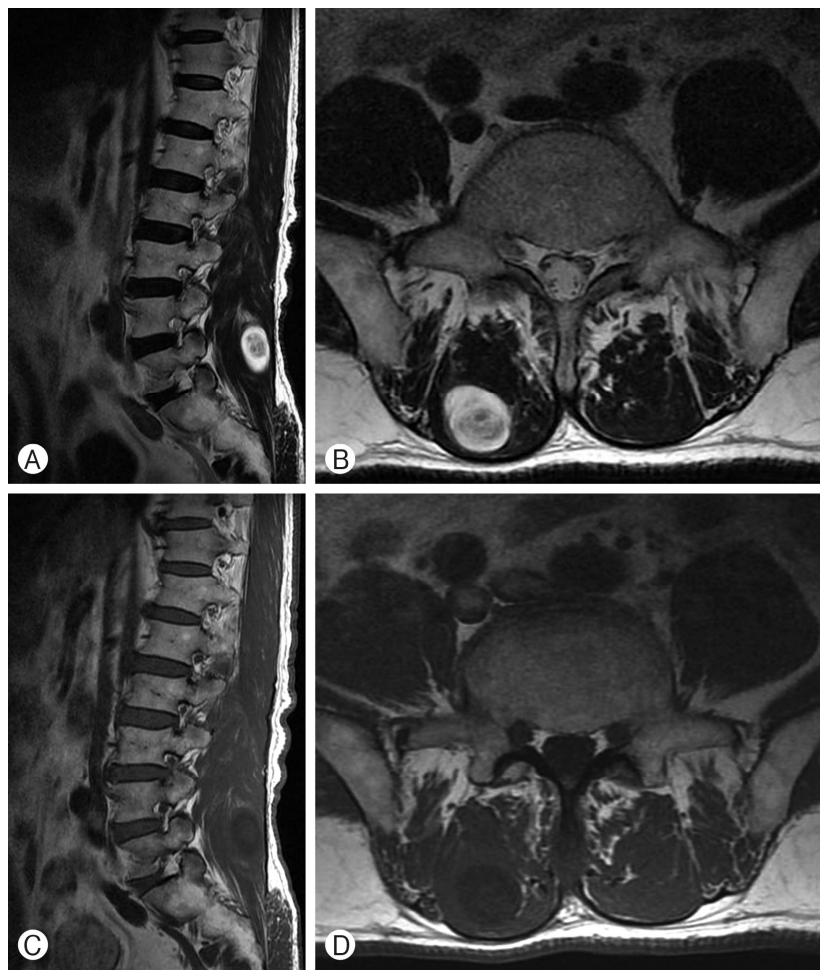

Fig. 1. Magnetic resonance imaging scan reveals an oval-shaped mass with high signal intensity (SI) and central intermediate SI foci on T2-weighted image; (A) sagittal, (B) axial. Heterogeneous intermediate $\mathrm{SI}$ on $\mathrm{Tl}$; (C) sagittal, (D) axial. 
clear margins in the multifidus muscles and could be distinguished from the surrounding structures (Fig. 2). Microscopically, the tumor was composed of compact spindle cells (Antoni A) alternating with loosely arranged foci (Antoni B) (Fig. 3A). Immunohistochemistry showed diffuse, high S-100 protein expression, and S-100 immunohistochemistry was positive (Fig. 3B). Histopathological analysis identified the mass as a schwannoma.

There were no immediate neurological deficits following surgery, and the patient was discharged with decreased tenderness.

\section{DISCUSSION}

The most common benign soft-tissue masses are lipomas and their variants. Moreover, fibrous histiocytoma, nodular fasciitis, hemangioma, and schwannoma may occur in soft tissues, in this order of frequency ${ }^{4,11)}$.

Schwannomas are benign tumors that arise from nerve sheath cells of peripheral nerves but rarely from within the muscle tissue. Less than $1 \%$ become malignant and transform into a form of cancer known as neurofibrosacroma ${ }^{11)}$. Progressive rapid growth with pain and cystic changes may be signs of malignant transformation ${ }^{2)}$. Although schwannomas can occur anywhere in the body, typical schwannoma cases are observed in the head and neck, with rates of $25 \%$ and $45 \%$, respectively ${ }^{11)}$. About 3 -quarters of benign solitary schwannomas are located in the upper limb arising from the brachial plexus or other nerves within the posterior triangle of the neck ${ }^{5}$. Complete surgical resection is the treatment of choice anywhere within
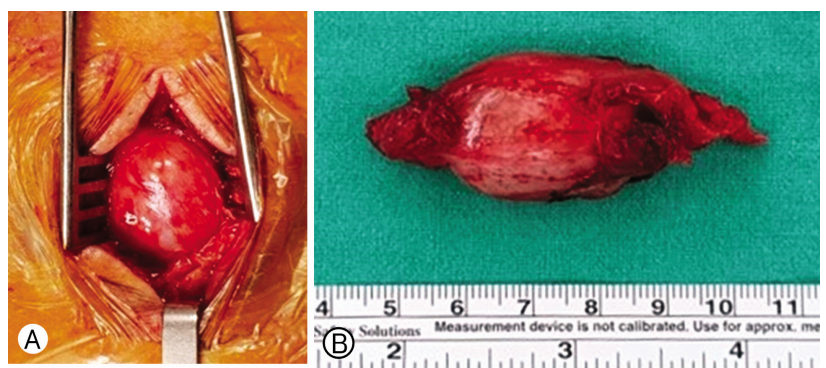

Fig. 2. Operative photographs show an oval shaped, well-encapsulated tumor (A) measuring approximately $2.0 \times 2.0 \times 3.5 \mathrm{~cm}(\mathrm{~B})$.
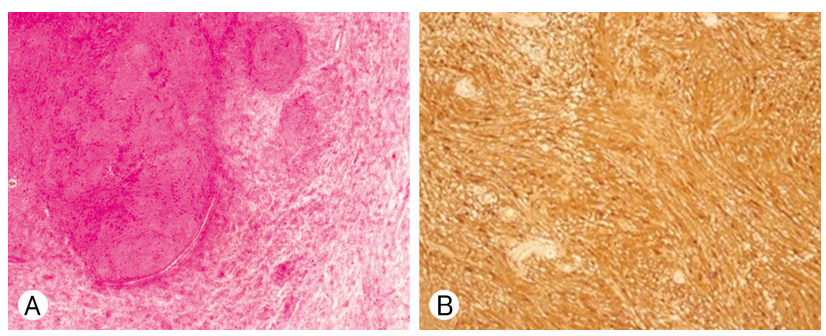

Fig. 3. Histopathologically, the biphasic areas are noted as compact areas of spindle cells (Antoni A tissue), alternating with loosely arranged foci (Antoni B) (A), and S-100 protein expression (B). the spinal axis ${ }^{6}$.

Intramuscular schwannomas originate from a small nerve branch within the muscle, and they are uncommonly associated with neurological symptoms including radiating pain, numbness, or motor weakness ${ }^{8)}$. In cases of the psoas muscle involving schwannoma, abdominal pain and femoral nerve neuropathy may occur ${ }^{1,3,7)}$. In our case, intramuscular schwannoma occurred in the multifidus muscle at the $\mathrm{L} 5$ level. Our patient complained of tenderness without radiating pain and lower extremity weakness.

The dominant nerve of the paraspinal muscles is the lumbar dorsal ramus nerve, a branch of the spinal nerve that forms from the dorsal root. The dorsal ramus nerve innervates a narrow strip of the skin and muscle along the back. This distribution of the nerve is of prime importance for the diagnosis of a nerve sheath tumor ${ }^{4,11)}$.

In MRIs', the lesion may have intermediate signal intensity on T1-WI, and heterogeneous on $\mathrm{T} 2-\mathrm{WI}^{2)}$. After contrast enhancement, the cystic lesion and well-enhanced peripheral or solid mass lesions are clearly distinct from each other. In the split fat sign, entering and exiting nerve in low-signal, and a thin hyperintense rim were detected ${ }^{10)}$. Low signal margin and fascicular sign on MRI are strong suggestive findings for diagnosing schwannoma $^{10)}$. The target sign was observed on T2-WI. This sign corresponded to the Antoni A area centrally and hyperintense peripheral rim similar to the Antoni B area. The MRI remains an important diagnostic tool; diffusion-weighted MRI may be used to differentiate benign paraspinal schwannomas from those of malignant nature" ${ }^{9)}$ The typical signs of schwannomas are more common in major nerve schwannomas than intramuscular schwannomas ${ }^{12)}$.

The Antoni A area is composed of compact spindle cells arranged in short bundles or interlacing fascicles. The Antoni B area is highly vascularized, less cellular, and lacks distinctive architectural structures ${ }^{1)}$. Immunohistochemically, strong S-100 protein expression was observed.

There was possibly only one case of dorsal ramus nerve schwannoma within the erector spinae muscles ${ }^{4}$. This case report describes a rare paraspinal schwannoma located in the multifidus muscle.

\section{CONCLUSION}

When a patient has a palpable, intramuscular back mass, an MRI should be considered. Although rare, schwannoma should be included in the differential diagnosis of a well-defined, oval-shaped soft tissue mass arising within the paraspinal muscles.

\section{CONFLICTS OF INTEREST}

No potential conflict of interest relevant to this article was reported. 


\section{REFERENCES}

1. D'Silva KJ, Dwivedi AJ, Barnwell JM: Schwannoma of the psoas major muscle presenting with abdominal and back pain. Dig Dis Sci 48:1619-1621, 2003

2. Dorsi MJ, Belzberg AJ: Paraspinal nerve sheath tumors. Neurosurg Clin N Am 15:217-222, vii, 2004

3. Hsu YC, Shih YY, Gao HW, Huang GS: Intramuscular schwannoma arising from the psoas muscle presenting with femoral nerve neuropathy. South Med J 103:477-479, 2010

4. Kim JH, Cho TG, Kim CH, Moon JG, Lee HK: Erector spinal muscular schwannoma of the dorsal ramus nerve: A case report. Korean J Spine 12:204-206, 2015

5. Knight DM, Birch R, Pringle J: Benign solitary schwannomas: a review of 234 cases. J Bone Joint Surg Br 89:382-387, 2007

6. Lee S, Srikantha U: Surgical management of giant lumbar extradural schwannoma: Report of 3 cases. Int J Spine Surg 9:18,
2015

7. Muramatsu K, Ihara K, Yoshida Y, Taguchi T: Intramuscular schwannoma arising from the psoas major muscle. Clin Neurol Neurosurg 110:532-533, 2008

8. Nishio J, Ueki T, Naito M: Intramuscular schwannoma of the musculocutaneous nerve: An uncommon clinical presentation. Exp Ther Med 6:164-166, 2013

9. Razek A, Ashmalla GA: Assessment of paraspinal neurogenic tumors with diffusion-weighted MR imaging. Eur Spine J 27: 841-846, 2018

10. Salunke AA, Chen Y, Tan JH, Chen X, Foo TL, Gartner LE, et al.: Intramuscular schwannoma: clinical and magnetic resonance imaging features. Singapore Med J 56:555-557, 2015

11. Shah KA, Shah AC: Paraspinal schwannoma of dorsal ramus nerve: A case report. J Clin Orthop Trauma 9:S1-S4, 2018

12. Shimose S, Sugita T, Kubo T, Matsuo T, Nobuto H, Tanaka K, et al.: Major-nerve schwannomas versus intramuscular schwannomas. Acta Radiol 48:672-677, 2007 